\title{
Desarrollo de proyectos para la formación de la competencia intercultural por estudiantes de medicina
}

\author{
Juan Manuel Muñoz-Cano, Teresita Maldonado-Salazar, Juan Bello
}

Objetivo. Contribuir a la formación de la competencia intercultural en estudiantes de medicina, considerada esencial para el desempeño del médico.

Sujetos y métodos. Para generar estrategias que permitan desarrollar la competencia intercultural en la educación médica, se realizó una intervención educativa con 44 estudiantes de una universidad del sureste de México en el verano de 2010. Se trabajó en base a proyectos de tipo de problemática. El producto debería ser un diagnóstico de comunidad que considerara las diferencias culturales.

Resultados. La experiencia la finalizaron el $56 \%$ de los participantes. De ellos, la mitad hizo exploraciones de percepción de la salud en sus prácticas comunitarias en otras asignaturas. El análisis de oraciones clave de sus informes permitió evaluar el cambio conceptual. Al aplicar el cuestionario de Ramsden se encontró que los estudiantes manifestaron haber mejorado sus habilidades genéricas (resolver problemas, realizar análisis, trabajar con otros estudiantes, trabajar en equipo, trabajar en la incertidumbre, planificar el propio trabajo) y que tuvieron muy buena percepción del curso.

Conclusiones. Aunque persistieron las dificultades para identificar las barreras y los dilemas culturales, en general mejoraron notablemente, así como en el manejo de las bibliotecas virtuales.

Palabras clave. Aprendizaje basado en proyectos. Educación médica. Interculturalidad.

\section{Development projects for the training of intercultural competence by medical students}

Aim. To contribute to the formation of the intercultural competence in medical students, essential for the medical performance.

Subjects and methods. To generate strategies to develop intercultural competence in medical students, an educational research was conducted with 44 students at a university in the Southeast of Mexico in the summer of 2010. It was worked with projects based on type of problem. The product should be a community intervention to consider cultural differences.

Results. $56 \%$ of the participants ended the study, of these, half had scans perception of health in their communities. The analysis was made by mean of key sentences of their reports to assess the conceptual change. Applying Ramsden's questionnaire found that students said they has improved their generic skills (problem solving, analysis, working with other students, teamwork, job uncertainty, plan own work) and they had very good perception of the course.

Conclusions. While overall they skills and middle management of virtual libraries improved greatly, difficulties remained to identify barriers and cultural dilemmas.

Key words. Interculturalism. Medical education. Project-based learning.

\section{Introducción}

La competencia intercultural tiene como fundamento una práctica de la medicina donde la relación médico-paciente se construye de manera horizontal. Ambos, como seres concretos, tienen una identidad conformada por el sexo, la cultura étnica, la clase social, la edad, la preferencia sexual, la asociación gremial y otros factores que la moldean; por esto, debe configurarse una relación intercultural en la comprensión de 'cómo' enferma la gente que ha de fomentarse desde el proceso escolar de la educación médica [1]. La competencia intercultural es un mecanismo propicio para aumentar la calidad de la atención médica, sobre todo a la población de menos ingresos o más desprotegida $[2,3]$, por lo que los planes de estudio de medicina de países desarrollados como Estados Unidos, Canadá, Reino Unido, Australia y Nueva Zelanda incluyen programas enfocados hacia la interculturalidad [4-7], con
Universidad Juárez Autónoma de Tabasco; Villahermosa, Tabasco (J.M. Muñoz-Cano). Universidad Pedagógica Nacional (T. MaldonadoSalazar, J. Bello). Universidad Nacional Autónoma de México (J. Bello). Secretaría del Medio Ambiente y Recursos Naturales; Centro de Educación y Capacitación Ambiental (T. Maldonado-Salazar). México DF, México.

Correspondencia:

Prof. Juan Manuel Muñoz Cano. Centro de Investigación. División Académica de Ciencias de la Salud. Universidad Juárez Autónoma de Tabasco. Avda. Méndez, 2838-A. CP 86150. Villahermosa, Tabasco, México.

E-mail:

juan.munoz@ujat.mx

Conflicto de intereses: No declarado.

Competing interests: None declared.

(C) 2014 FEM 
diferentes niveles de concreción para superar las barreras culturales.

Las barreras no sólo se refieren a cuestiones relativas a diferentes idiomas, son los obstáculos al proceso de cambio social y a las modificaciones culturales necesarias para disminuir el impacto de las enfermedades en la salud pública, tanto para comunidades aisladas, habitadas por campesinos indígenas pobres [8], como en las mismas poblaciones urbanas culturalmente heterogéneas $[3,9]$. Formar la competencia intercultural como objetivo longitudinal del proceso de educación médica debe ser una de las metas a medio plazo en los procesos de educación médica de países que, como México, tienen una gran diversidad cultural. La competencia intercultural es un paradigma emergente en el sistema educativo [10] y la formación de un profesional competente requiere con urgencia aproximaciones innovadoras para el logro de este propósito.

\section{Competencia intercultural}

El marco teórico del enfoque intercultural -como práctica para el fortalecimiento de los servicios del Sistema Nacional de Salud de México- tiene referentes de 'las teorías de la comunicación, el indigenismo y la teoría de sistemas' [11]. Para la Secretaría de Salud de México, el profesional culturalmente competente ha desarrollado capacidades de negociación y comunicación, 'con el fomento de actitudes de respeto, tolerancia, diálogo y enriquecimiento mutuo, constatando que la verdad es plural y relativa, y que la diversidad puede ser fuente de riqueza' [11]. Aunque su contexto de desempeño primordial son los pueblos indígenas, en esta perspectiva deben considerarse grupos diferentes que cohabitan en una sola comunidad. La base de la sociedad pluricultural [12], para lo cual se requieren enfoques metodológicos complejos como el que considera la metacognición, permite el desempeño de una medicina donde a las personas se las trata como a 'ellas' quieren que se les trate.

$\mathrm{El}$ aprendizaje en la complejidad requiere estrategias integradoras, una de las cuales es el aprendizaje basado en proyectos, que no se emplea en la educación médica, si bien se ha aplicado el aprendizaje basado en problemas. El desarrollo del aprendizaje basado en proyectos se establece como un tema centrado hacia una problemática de mayor amplitud de las situaciones que se emplean para el aprendizaje basado en problemas [13-15]. La diferencia entre proyectos y problemas es la amplitud a la que se dirigen, ya que ambos son complejos. Los proyectos se dirigen a comunidades, grupos huma- nos o familias, y los problemas, en medicina, son relativos a pacientes individuales.

El aprendizaje basado en proyectos permite la construcción de las capacidades necesarias para el enfoque intercultural porque promueve una conciencia de respeto de otras culturas, lenguas y personas, desarrollar empatía por la gente y proveer una herramienta y una metodología para aprender cosas nuevas de modo eficaz [16], algo necesario en la construcción de las capacidades de aprender a resolver problemas, aprender de manera autónoma y, específicamente, la visión intercultural [17]. También es una estrategia que permite configurarse en una actividad de investigación formativa, que es la base de intervenciones de mercadotecnia en salud [18]. El estudiante, al realizar un proyecto en el que identifica causas y efectos como variables independientes y dependientes, puede definir los conceptos que necesita construir, los textos que necesita revisar para mejorar su comprensión de un fenómeno en su contexto con el fin de generar pensamiento crítico [19]. La construcción de la interculturalidad ofrece mayores dificultades para su construcción que otras competencias no sólo porque se requiere flexibilidad y creatividad, sino aceptación de los otros [20].

A pesar de que la competencia intercultural ha tenido un importante desarrollo conceptual y metodológico en los últimos años [1,6,17], muchos enfoques curriculares aplicados son limitados e insuficientes [21]; esto sucede aún más tímidamente en México, país con una amplia diversidad cultural. Por esta razón se realizó esta investigación educativa en base a las preguntas: ¿cómo ayudar a los estudiantes a desarrollar capacidades para identificar problemáticas en distintos contextos sociales?, ¿cómo enseñar a los estudiantes a identificar las causas en las que podrían hacer énfasis en actividades de atención de prevención y promoción a la salud en el primer nivel de atención?, y ¿cómo facilitar que los estudiantes perciban las diferencias culturales entre ellos y los pacientes con el fin de superar obstáculos para la comunicación?

\section{Sujetos y métodos}

El propósito fue contribuir a la formación de la competencia intercultural en estudiantes de medicina, para lo cual se realizó una investigación cualitativa de tipo participante.

\section{Participantes}

El curso se realizó con 44 estudiantes de medicina 
Tabla I. Matriz de evaluación continua.

\begin{tabular}{|c|c|c|c|c|}
\hline & Nivel 4 & Nivel 3 & Nivel 2 & Nivel 1 \\
\hline $\begin{array}{l}\text { Identificación de } \\
\text { la barrera cultural }\end{array}$ & $\begin{array}{l}\text { Propone acciones para remover } \\
\text { barreras en varios ámbitos }\end{array}$ & $\begin{array}{l}\text { Propone acciones para remover } \\
\text { barreras en alguno de los ámbitos }\end{array}$ & $\begin{array}{l}\text { Menciona los ámbitos } \\
\text { sin proponer acciones }\end{array}$ & $\begin{array}{l}\text { No identifica las } \\
\text { barreras culturales }\end{array}$ \\
\hline $\begin{array}{l}\text { Identificación de la aplicación } \\
\text { de la interculturalidad a un } \\
\text { problema de salud }\end{array}$ & $\begin{array}{l}\text { Determina los criterios } \\
\text { para que las acciones } \\
\text { mejoren la calidad del servicio }\end{array}$ & $\begin{array}{l}\text { Identifica los criterios a considerar } \\
\text { en la determinación de acciones }\end{array}$ & $\begin{array}{l}\text { Identifica contenidos de inter- } \\
\text { culturalidad que deben integrarse } \\
\text { en las unidades de atención }\end{array}$ & $\begin{array}{l}\text { Se limita a transcribir fragmentos } \\
\text { de los textos que consulta }\end{array}$ \\
\hline $\begin{array}{l}\text { Identificación del } \\
\text { dilema cultural }\end{array}$ & $\begin{array}{l}\text { Identifica la cosmovisión y las } \\
\text { explicaciones de los problemas } \\
\text { de salud de la población a atender }\end{array}$ & $\begin{array}{l}\text { Toma una posición respecto } \\
\text { del problema, pero no } \\
\text { identifica otras facetas de éste }\end{array}$ & $\begin{array}{l}\text { Elabora definiciones relacionadas } \\
\text { con el problema, pero no } \\
\text { identifica diversas explicaciones }\end{array}$ & $\begin{array}{l}\text { Mantiene una posición } \\
\text { simplista del problema }\end{array}$ \\
\hline Búsqueda de información & $\begin{array}{l}\text { La información proviene de } \\
\text { fuentes primarias o de artículos } \\
\text { científicos y los cita correctamente }\end{array}$ & $\begin{array}{l}\text { La información proviene de fuentes } \\
\text { primarias o artículos que la refieren, } \\
\text { pero no las cita correctamente }\end{array}$ & $\begin{array}{l}\text { Al menos la mitad de su } \\
\text { información proviene de fuentes } \\
\text { fiables, aunque falla al citarlas }\end{array}$ & $\begin{array}{l}\text { Cita de páginas } \\
\text { electrónicas sin calidad }\end{array}$ \\
\hline $\begin{array}{l}\text { Elaboración de conclusiones } \\
\text { y recomendaciones }\end{array}$ & $\begin{array}{l}\text { Sustenta las conclusiones } \\
\text { en el análisis de la } \\
\text { información consultada }\end{array}$ & $\begin{array}{l}\text { Escribe enunciados que } \\
\text { no expresan conclusiones y } \\
\text { recomendaciones puntuales }\end{array}$ & $\begin{array}{l}\text { Enuncia ideas inconexas } \\
\text { relacionados con el problema }\end{array}$ & $\begin{array}{l}\text { Sus enunciados no contienen } \\
\text { información relevante } \\
\text { respecto al problema }\end{array}$ \\
\hline Ortografía y gramática & El escrito muestra ilación & $\begin{array}{l}\text { Comete un máximo } \\
\text { de diez errores ortográficos }\end{array}$ & $\begin{array}{l}\text { Comete un error } \\
\text { ortográfico por línea }\end{array}$ & No sigue las reglas ortográficas \\
\hline
\end{tabular}

Se discutió con los estudiantes al inicio del curso, se empleó para retroalimentar los avances de los estudiantes, tanto en descripción de problemas como avances en sus proyectos. Elaboración propia.

distribuidos en dos grupos. Como se trataba de un programa flexible producto de la reestructuración curricular de la carrera de medicina realizada en 2006, los estudiantes podían inscribirse libremente en las asignaturas para las cuales tuvieran mayor interés inmediato. 'Medicina intercultural' formó parte de los cursos optativos, de cuatro créditos, que se ofertaron en el verano de 2010 con el fin de que tuvieran mayor avance curricular. De esa manera, los estudiantes tenían diferente número de semestres cursados. Los nombres de los estudiantes se codificaron con una letra y el número 1 o 2 de acuerdo con el grupo en el que se encontraban inscritos.

\section{Instrumentos de evaluación}

Se emplearon distintos instrumentos para evaluar los aspectos relevantes del curso: una matriz de evaluación para la retroalimentación, la escala de integración del texto para los informes de los estudiantes y el cuestionario de Ramsey para la evaluación del curso y el desempeño del docente.

\section{Matriz de evaluación}

Se construyó en base a seis categorías:

- Identificar la barrera cultural que se debe considerar para el desarrollo del proyecto.
- Considerar elementos de la interculturalidad en sus propuestas.

- Identificar el o los dilemas culturales que se deben afrontar para que se aplique la intervención educativa.

- Capacidad de identificar y analizar información.

- Elaboración de conclusiones y recomendaciones de la información.

- Ortografía y gramática de los informes.

Cada categoría tuvo cuatro niveles; por ejemplo, para la categoría 'identificación de la barrera intercultural', nivel 1 cuando no las identifica y nivel 4 si propone acciones para remover barreras en varios ámbitos (Tabla I).

\section{Análisis de los informes de los estudiantes}

Se realizó de acuerdo con la escala de integración del texto [22,23], con cuatro niveles:

- Texto transcrito: copiado de párrafos incluso con ligas y estilos de letra.

- Texto fragmentado: se identifica reproducción literal, parafraseo, tratamiento parcelado, no hay cierre.

- Integrado parcialmente: persisten el parafraseo y fallos en la vinculación entre los párrafos.

- Integrado: párrafos de transición entre las temá- 
Tabla II. Propuestas de los estudiantes antes y después de la intervención.

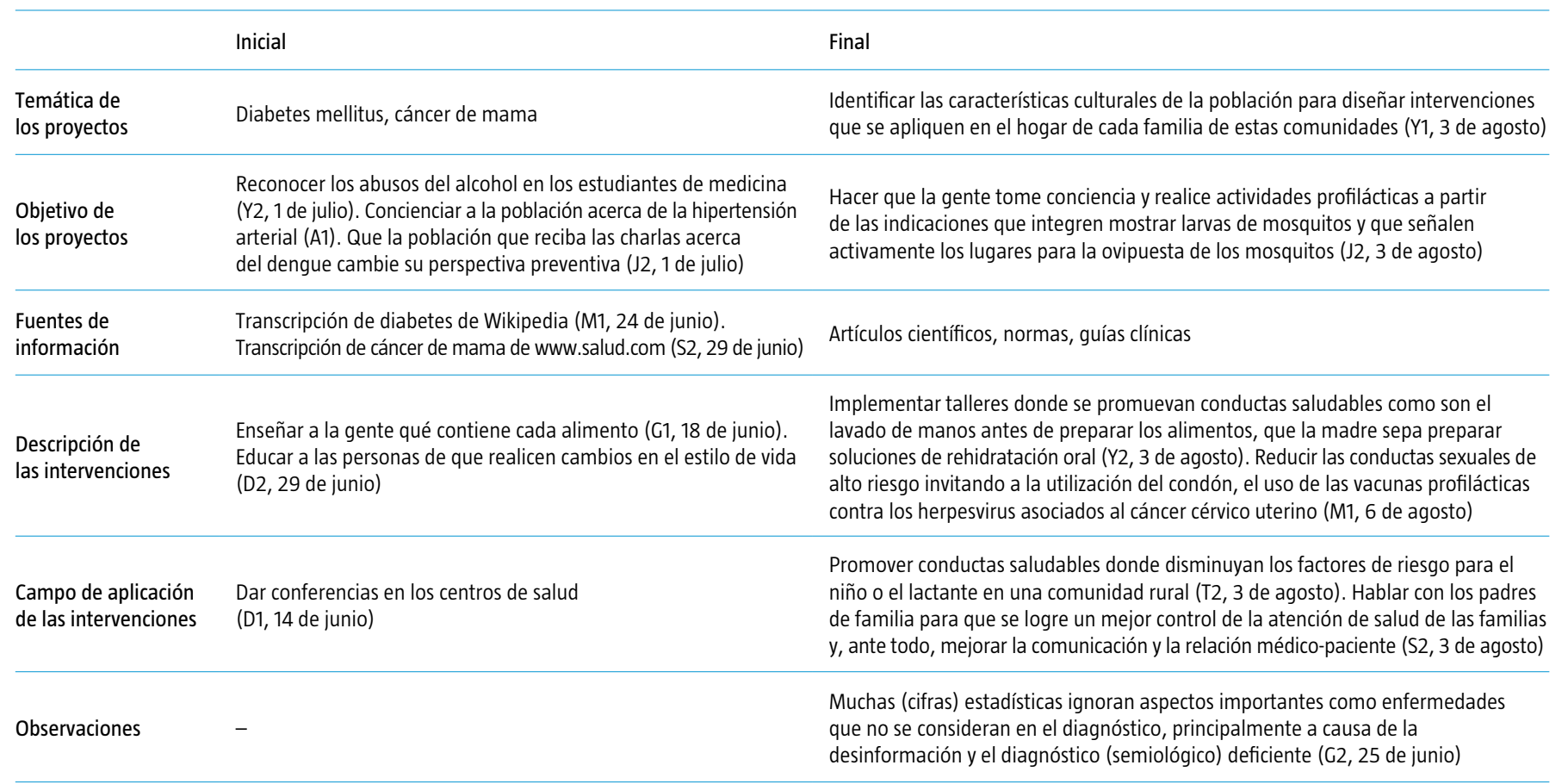

Comparación de las construcciones gramaticales de los estudiantes en sus propuestas iniciales y en trabajos cercanos al final del curso. Se observa un mayor análisis, por ejemplo en la diferencia entre tema y problema (categoría temática). Elaboración propia en base a los escritos de los estudiantes.

ticas, presencia de conectores, uso coherente de las referencias, jerarquización de los conceptos.

\section{Course Experience Questionnaire de Ramsden}

Es parte de los recursos pedagógicos de la UK Centre for Materials Education disponibles en línea en su sitio web [13]. El cuestionario se centra en lo que los estudiantes aprenden, sobre todo en las actividades del docente que fomentan el aprendizaje significativo. Las subescalas del cuestionario exploran actitudes e intenciones del profesor y son:

- Buena práctica docente.

- Metas y reglas claras.

- Prácticas de evaluación formativa apropiadas.

- Carga de trabajo apropiada.

- Desarrollo de habilidades genéricas (resolver problemas, realizar análisis, trabajar con otros estudiantes, trabajar en equipo, trabajar en la incertidumbre, planificar el propio trabajo).

- Percepción general del curso.

La escala del cuestionario va desde 'en desacuerdo' (1 punto) a 'completamente de acuerdo' (4 puntos). Tiene ítems con carga positiva, como 'el curso me ayudó a desarrollar mis habilidades', y con carga negativa, como 'sentí una gran presión para hacer las cosas correctamente'. Se realizó una prueba piloto del cuestionario con estudiantes de otros cursos con el fin de evaluar la traducción de los reactivos al castellano.

\section{Procedimiento}

El curso se estructuró en base a proyectos [19] del tipo de problemática [14] y se organizó durante el verano de 2010, del 21 de junio al 6 de agosto. Como producto del proyecto [24] se solicitó a los estudiantes el diseño de una intervención educativa para afrontar problemas de salud pública y tendría como eje la interculturalidad, especialmente en comunidades indígenas.

El docente responsable de la clase proporcionó a los estudiantes un número limitado de páginas de Internet con el fin de que no tuvieran dificultades por la gran cantidad de información existente en el ciberespacio. Los textos de la lista fueron primordialmente publicaciones de la Secretaría de Salud de México en su página web. Se recomendaron tam- 
bién organizaciones relacionadas con los derechos y las minorías en México que sustentan la ciudadanía multicultural: Comisión Nacional para el Desarrollo de los Pueblos Indígenas, Comisión Nacional de los Derechos Humanos, Consejo Nacional para Prevenir la Discriminación y el Instituto Nacional de las Mujeres.

La retroalimentación del curso se realizó en base a la evaluación formativa [25], con el propósito de identificar las necesidades de aprendizaje y ajustar la enseñanza adecuadamente, así como elaborar materiales, asesorías definidas o discusiones específicas. Para el diseño del curso se consideraron contenidos de diferentes asignaturas. El objetivo fue desarrollar capacidades para la elaboración de proyectos, resolución de problemas y trabajo en equipo, entre otras competencias transversales enfocadas hacia la construcción de la competencia intercultural del estudiante de medicina.

El objetivo de la evaluación final fue analizar conjuntamente con los estudiantes los resultados y el proceso realizado: errores, logros, dificultades, utilidad de la retroalimentación y sugerencias. La acreditación se obtuvo una vez cumplidos el $80 \%$ de los criterios especificados en la matriz de evaluación para el producto completo, o al menos todos los indicadores en el nivel 2 de la matriz de evaluación (Tabla I), y de acuerdo con esas puntuaciones obtuvieron su calificación.

\section{Tratamiento estadístico}

Se elaboró una base de datos con los resultados de los cuestionarios de Ramsden mediante el programa SPSS v. 15.0. Se efectuó una prueba de $\alpha$ de Cronbach para evaluar la consistencia interna de las respuestas y se obtuvo un valor de 0,7. Se realizó una prueba de medias \pm desviación estándar para las puntuaciones de cada pregunta del cuestionario. Se aplicó una prueba de análisis de componentes principales a los reactivos del cuestionario. Se realizó una prueba de correlación de Pearson para determinar la asociación entre las categorías de análisis y se consideró significativo cuando $p \leq 0,01$.

\section{Consideraciones éticas}

Los estudiantes que se inscribieron en el curso tuvieron la posibilidad de darse de baja de él sin que se contabilizara como asignatura no aprobada. En los registros de cuestionarios y opiniones acerca del curso se codificó a los participantes para que conservaran el anonimato. El estudio contó con la autorización de los comités de investigación y de ética de la
División Académica de Ciencias de la Salud (DACS) de la Universidad Juárez Autónoma de Tabasco.

\section{Resultados}

Los resultados de la intervención se presentan en dos componentes: los avances de los estudiantes en la construcción de la competencia a través de sus informes, y la evaluación de las actividades del equipo docente por los estudiantes y el análisis de estos resultados en la explicación de las dificultades de los estudiantes ante un proceso innovador.

\section{Productos de los proyectos}

Las propuestas de los estudiantes en la primera semana se limitaron a datos que se podrían encontrar rápidamente, por ejemplo, en el sitio web de la Secretaría de Salud de México, tales como prevalencia de diabetes mellitus tipo 2 , incidencia de neumonía en un grupo de edad o consumo de alcohol por estudiantes, aportaciones del tipo de lista de inventario. Aunque los estudiantes habían cursado asignaturas como Salud Pública y Epidemiología, sus propuestas para realizar intervenciones se limitaban al enfoque centrado en grupos cautivos, como personas en la sala de espera de los centros de salud, hospitales o grupos de estudiantes de medicina, en intervenciones de tipo expositivo. Se tuvo que reflexionar con ellos para que identificaran las tablas de morbilidad, mortalidad y altas hospitalarias como fuentes elementales de información para la construcción de problemas de salud, e integrar la información para la realización de un diagnóstico de la comunidad.

Durante las siguientes tres semanas se avanzó en la construcción de los contextos de aplicación de los problemas, pues se transitó de las participaciones con base en temas a identificar las causas de los problemas en el contexto social. Los estudiantes presentaron como suyas las respuestas que encontraron en la primera fuente que desplegaba su motor favorito de búsqueda. Se les invitó a que transitaran de la consulta de páginas poco fiables a las páginas oficiales, normas, guías clínicas y artículos primarios; esto se realizó en una aula en el centro de cómputo de la DACS. De esta manera se les acompañó en la identificación de información científica que ayudó a la identificación de causas y efectos, no simplemente agentes etiológicos, sino contextuales (Tabla II).

Se animó a los estudiantes a trabajar los objetivos del curso en concordancia con sus prácticas en 
Tabla III. Informes de los estudiantes acerca de sus intervenciones.

\begin{tabular}{|c|c|c|}
\hline $\begin{array}{l}\text { ¿Cuál es el problema } \\
\text { que percibí en la comunidad? }\end{array}$ & $\begin{array}{l}\text { ¿Cómo lo percibe la gente, } \\
\text { qué conductas realiza, qué piensa? }\end{array}$ & ¿Cómo lo resolví o puedo resolverlo? \\
\hline $\begin{array}{l}\text { Muchas de las mujeres aún tienen miedo } \\
\text { o las aflige que un médico las explore, } \\
\text { muchas veces porque el marido se lo prohíbe }\end{array}$ & $\begin{array}{l}\text { Al principio llegan muy pocas, no le toman } \\
\text { mucha importancia, no te quieren contar } \\
\text { todos sus problemas a menos que uno les } \\
\text { pregunte. Piensan que uno no sabe y sólo } \\
\text { se viene a experimentar con ellas }\end{array}$ & $\begin{array}{l}\text { Las cité en un centro de salud de su comunidad para darles una charla e } \\
\text { información acerca de enfermedades, les dije que fueran con sus esposos e hijas. } \\
\text { Luego, por medio de un maniquí, les enseñe cómo deben explorarse, cuándo y } \\
\text { dónde, les dije que lo practicaran en su hogar. Cuando entendieron cómo hacerlo } \\
\text { las invité a que acudieran al centro de salud (G1, } 5 \text { de agosto) }\end{array}$ \\
\hline $\begin{array}{l}\text { Percibí la forma de pensar negativa } \\
\text { de los padres acerca del cuidado que } \\
\text { deben llevar ellos por padecer diabetes } \\
\text { mellitus tipo } 2 \text { y el de los hijos por ser } \\
\text { propensos a padecerla }\end{array}$ & $\begin{array}{l}\text { Decían que era innecesario cambiar su } \\
\text { estilo de vida y sus costumbres ya que } \\
\text { no han tenido consecuencias por la forma } \\
\text { de vida que llevan; recalcaron que era } \\
\text { innecesario y que esa forma de vida } \\
\text { no afecta a los niños ya que no sufren } \\
\text { riesgo alguno }\end{array}$ & $\begin{array}{l}\text { Resolví el problema explicándoles y enseñándoles ejemplos de las complicaciones } \\
\text { que ocasionan los hábitos incorrectos de su vida; con los talleres, los padres } \\
\text { accedieron a practicar las soluciones presentadas considerando nuevas ideas } \\
\text { para que los niños entendieran el cuidado de una alimentación sana y la práctica } \\
\text { del deporte. Los padres entendieron la importancia de un buen cuidado y se } \\
\text { mostraron muy optimistas y participativos en las dinámicas. Los niños, a pesar } \\
\text { de ser pequeños, entendieron el mensaje y cambiaron hábitos que favorecieron } \\
\text { un estilo de vida saludable (R2, } 4 \text { de agosto) }\end{array}$ \\
\hline
\end{tabular}

Los estudiantes lograron identificar una necesidad de aprendizaje y elaboraron textos más integrados. Esto se encuentra en la descripción de los problemas de las comunidades por los estudiantes y en la elaboración de acciones de acuerdo con la percepción de la gente, así como en la transcripción de sus conceptos al lenguaje cotidiano para integrarse en la comunidad. Elaboración propia en base a los informes de los estudiantes.

el primer nivel de atención. Las actividades con distintas estrategias, realizadas por 12 de los 22 estudiantes que aún permanecían en el curso, les permitió avanzar más en la identificación del contexto cultural y su impacto en la percepción de la salud, la enfermedad, la atención médica y las características necesarias de una intervención educativa, ya que dejaron de mencionar 'explicar' o 'informar'. En esta etapa final mencionaron más la elaboración de talleres, mostrar cómo, dar ejemplos, y seis de los informes corresponden a observaciones de calidad, con un mayor manejo conceptual y una mejor percepción del contexto de aplicación, lo cual es indicativo de una mejor construcción de sus textos ( Tablas I y III).

El equipo docente evaluó los informes que los estudiantes enviaron durante el curso de acuerdo con la matriz de evaluación. Los resultados concuerdan en la mayor parte de las casillas, aunque donde menos correspondencia se observa es en el manejo de las bibliotecas virtuales y en la identificación de las barreras y los dilemas culturales. Los estudiantes realizaron un ejercicio de autoevaluación, y aunque hay puntos discordantes con la evaluación del equipo docente, tienen mayor capacidad en identificación de la barrera cultural $(2,5 \pm$
$1,0)$, aunque no lo hicieron con los elementos de la interculturalidad ni los dilemas que se han de trabajar en las propuestas educativas en el contexto real. Lograron la elaboración de conclusiones y recomendaciones con base a un mejor, aunque no óptimo, manejo de información $(2,4 \pm 1,3)$. Mejoraron mucho en el cuidado del idioma de sus informes $(3,7 \pm 0,5)$.

\section{Evaluación de la intervención por los estudiantes}

Con el fin de que los estudiantes evaluaran el curso, se aplicó el cuestionario de Ramsden como actividad de cierre. La prueba de medias \pm desviación estándar con rango entre 1 y 4 puntos de acuerdo con la escala descrita mostró una puntuación elevada para la mayoría de las preguntas como resultado de la evaluación de los componentes principales. Esto determinó que las medias se analizaran con la suma de los resultados de las preguntas por categoría. El promedio más alto se obtuvo en la categoría de desarrollo de habilidades genéricas $(3,28 \pm 0,44)$ y el más bajo, en la de prácticas de evaluación formativa $(2,52 \pm 0,34)$, así como en carga de trabajo apropiado $(2,58 \pm 0,47)$, donde hay tres y dos preguntas con carga negativa. De éstas, la pregunta 19 ('el 
equipo docente me hizo muchas preguntas de datos y conceptos') obtuvo una puntuación alta, una media de 3,5 , ya que parte de la estrategia consistió en realizar muchas preguntas para que transitaran a zonas de menos comodidad conceptual.

$\mathrm{Al}$ realizar la correlación entre las categorías se encuentra la alta significación de la percepción general del curso con las prácticas docentes $(p=0,01)$, así como el desarrollo de las habilidades genéricas $(p=0,01)$. En este análisis se hizo una categoría con las preguntas con carga negativa, las que se correlacionan con las prácticas de evaluación formativa $(p=0,01)$ y la carga de trabajo $(p=0,01)$ (Tabla IV). La escala de tipo Likert no permitió conocer lo que los estudiantes opinan en algunos puntos, por ello se les animó a que escribieran lo que consideraran fuera de una pregunta o en términos amplios. Así, expresaron: 'al comenzar el curso no imaginaba siquiera de qué trataría. Al comienzo fue difícil pues no captaba la idea del profesor. Con el tiempo, las charlas y la retroalimentación se hicieron más interesantes y me vi más involucrado en la clase' (A1, 6 de agosto); 'la carga de trabajo fue un poco alta ya que teníamos que leer muchas cosas, no sólo un artículo, sino de la problemática de una enfermedad' (G1, 6 de agosto); 'me ayudó a ver las cosas de otra manera, en la escuela sólo son exámenes y calificaciones, tu mente sólo se enfoca en ello' (Y2, 6 de agosto).

Ya que la primera meta era formar capacidades personales, no hubo trabajo en equipos. Aunque los estudiantes no perciben el trabajo colaborativo en el aula como tal, se obtuvo una regular asistencia, así como observaciones a los proyectos y discusiones conceptuales que ellos tenían que construir. A este respecto, los estudiantes mencionaron: 'el trabajo consistía en saber buscar información y aprender cosas nuevas' (B1, 6 de agosto). El cuestionario de Ramsden evaluó la satisfacción de los estudiantes, y esto se reflejó en la puntuación $(3,58$ $\pm 0,50$ en el criterio de percepción general del curso) y en observaciones como: 'cambié mi perspectiva, mi forma de estudiar, investigar y de ver las cosas; diferente a como solía hacerlo' (D1, 6 de agosto); 'me gustó mucho, aprendí a comprender e identificar mejor los problemas y a darles una solución' (E1, 6 de agosto).

\section{Discusión}

Como parte de un proceso de reconfiguración de la educación médica, se realizó una intervención en un grupo de 44 estudiantes para desarrollar la com-
Tabla IV. Correlaciones entre las categorías del cuestionario de Ramsden.

\begin{tabular}{|c|c|c|c|c|c|c|}
\hline & 1 & 2 & 3 & 4 & 5 & 6 \\
\hline \multicolumn{7}{|l|}{ 1. Metas y reglas claras } \\
\hline 2. Buena práctica docente & 0,131 & & & & & \\
\hline $\begin{array}{l}\text { 3. Prácticas de evaluación } \\
\text { formativa apropiadas }\end{array}$ & 0,045 & 0,004 & & & & \\
\hline 4. Carga de trabajo apropiada & $-0,223$ & $-0,012$ & 0,074 & & & \\
\hline 5. Desarrollo de habilidades genéricas & 0,303 & $0,715^{b}$ & $-0,126$ & $-0,099$ & & \\
\hline 6. Percepción general del curso & 0,225 & $0,645^{b}$ & $-0,011$ & $-0,122$ & $0,543^{b}$ & \\
\hline $\begin{array}{l}\text { 7. Puntuación de las preguntas } \\
\text { con carga negativa }\end{array}$ & 0,091 & $-0,452^{a}$ & $0,482^{a}$ & $0,553^{b}$ & $-0,276$ & $-0,380$ \\
\hline
\end{tabular}

Resultados de la prueba de Pearson. Para elaborar la tabla se agruparon los resultados de cada categoría del cuestionario. Se creó una categoría con los reactivos con puntuación negativa. ${ }^{a}$ Correlación significativa al nivel 0,$05 ;$ b Correlación significativa al nivel 0,01. Elaboración propia.

petencia intercultural. Se hizo énfasis en la capacidad de negociar e interactuar, la capacidad de comunicación con grupos culturalmente diversos, hablantes en idiomas diferentes $[1,26]$, y la percepción de que la ciencia y la verdad son construcciones sociales. El eje primordial fue la elaboración de proyectos educativos donde los estudiantes integraron conceptos en la aplicación de información para resolver problemas de salud en comunidades con características culturales diferentes de las del médico, lo cual es un proceso innovador en esta universidad. Con el método empleado -basado en proyectos-, el proceso de aprender no se resuelve de la manera simplista como pretende la escuela ordinaria, lo que produce malestar en los estudiantes, ya que las preguntas con puntuación negativa se asocian con las prácticas de evaluación y la carga de trabajo (Tabla IV). No es suficiente la información -ni el conocimiento descontextualizado, pensado de manera homogeneizante [27], muchas veces opuesto a la gente que necesita educarse- para generar cambios conductuales que se reflejen en el tránsito hacia estilos de vida más saludables. Esto se refleja en que la experiencia sólo la terminaron el $56 \%$ de los inscritos. Este proceso, que puede facilitar cambios sociales, requiere el diseño de intervenciones innovadoras. La diferencia fundamental es que 'elabora un escenario de acción examinando las certezas y las incertidumbres, probabilidades, improbabilidades. El escenario puede y debe ser modificado según la información recogida, los aza- 
res, contratiempos u oportunidades encontradas en el camino' [28]. Por ello se emplearon diferentes instrumentos para evaluar los avances y las percepciones de los estudiantes (Tablas I a IV).

De los estudiantes que finalizaron, el 27\% lograron estructurar intervenciones donde se identificaran barreras interculturales. Aunque los estudiantes habían cursado asignaturas del área sociomédica, no se reflejó en la elaboración de sus primeras aproximaciones, e incluso al final, las otras culturas no aparecen en lo que escribieron. Esto es irónico en un país donde la diversidad cultural es alta y los mismos estudiantes provienen de localidades del interior del estado donde hay población indígena. Esto se encuentra en el análisis de los informes, donde el equipo docente halló deficiencias de los estudiantes en la incorporación de elementos de la interculturalidad y la identificación del dilema cultural.

Por otra parte, la idea general de estos estudiantes antes de la intervención es que para educar a las personas basta con un discurso, el mismo que se encuentra en el común de las intervenciones. Esta posición no considera la complejidad de las personas sencillas, y que la empatía, la aceptación y la negociación son vitales para un proceso de recuperación y conservación de la salud y el bienestar $[4,8,11]$. Esto constituye una limitación para el desarrollo de este proceso de atención en el primer nivel, ya que las intervenciones exitosas consideran 'diversos sectores e identifican a los actores involucrados en cada acción clave para la prevención' [29], capacidad que no lograban satisfactoriamente en el inicio ni siquiera los estudiantes con ocho semestres cursados de la licenciatura, y sólo lo hizo el $27 \%$ al final del curso.

Es significativo que abandonaran el $44 \%$ de los inscritos en este curso de verano. Prefieren asistir a los cursos tradicionales en los que las actividades se centran en la explicación y la exposición de contenidos temáticos de acuerdo con la secuencia de los programas de asignatura. Esto es un problema que se ha notado en intervenciones similares en esta dependencia educativa [9]; sin embargo, como en otras experiencias innovadoras en esta universidad, los estudiantes que la finalizaron se muestran satisfechos con la metodología y los resultados (Tablas II y III). En el aspecto de comunicación, no se puede afirmar que ya puedan conducir proyectos de investigación formativa o educación participativa necesarios para la solución de problemas de salud [18], aunque elaboraron una propuesta y determinaron sus asombros una vez que intentaron realizar la intervención en un nivel limitado, lo cual es parte de una toma de conciencia de sus propias represen- taciones y conceptualiza sus esquemas de conocimiento [28]. Esto sólo es una primera aproximación a este tipo de trabajo, que se encuentra en observaciones como: 'al principio no lograba comprender muy bien las metas ni lo que es interculturalidad' (A1, 6 de agosto).

\section{Bibliografía}

1. Bleszynska KM. Constructing intercultural education. Intercultural Education 2008; 19: 537-45.

2. ABC Cardiovascular Disparities Center. Medscape Family Medicine Education, Association of Black Cardiologist, 2011. URL: http://www.medscape.org/sites/advances/abc-cardiodisparity?src=0_mp_cmenl_0.

3. Montero-Mendoza E. Percepción de los habitantes indígenas de áreas rurales respecto al primer nivel de atención médica. El caso del sureste de Veracruz, México. Salud Colectiva 2011; 7: 73-86.

4. Dogra N, Reitmanova S, Carter-Pokras O. Teaching cultural diversity: current status in UK, US, and Canadian Medical Schools. JGIM 2010; 25 (Suppl 2): 164-8.

5. Gillard S, Benson J, Silverman J. Teaching and assessment of explanation and planning in medical schools in the United Kingdom: cross sectional questionnaire survey. Med Teach 2009; 31: 328-31.

6. Lie DA, Boker J, Crandall S, DeGannes CN, Elliot D, Henderson P, et al. Revising the Tool for Assessing Cultural Competence Training (TACCT) for curriculum evaluation: findings derived from seven US schools and expert consensus. Med Educ Online, 2008; 13: 1-11.

7. Seeleman C, Suurmon J, Stronks K. Cross-cultural medical education. Med Educ 2009; 43: 229-37.

8. Berenzon-Gorn S, Ito-Sugiyama E, Vargas-Guadarrama LA. Enfermedades y padeceres por los que se recurre a terapeutas tradicionales de la Ciudad de México. Salud Publica Mex 2006; 48: 45-56.

9. Muñoz-Cano JM. Experiencia metacompleja para la construcción de un modelo de diagnóstico médico por estudiantes. Educ Med 2012; 15: 89-94.

10. Gómez-Zermeño M. Competencias interculturales en instructores comunitarios que brindan servicio a la población indígena del estado de Chiapas. Rev Elec Invest Educ 2010. URL: http://redie.uabc.mx/vol12no1/contenido-gomezzermeno.html.

11. Secretaría de Salud. Interculturalidad en salud. Experiencias y aportes para el fortalecimiento de los servicios de salud. México DF: Secretaría de Salud; 2008.

12. Sartori G. Pluralismo, multiculturalismo y extranjeros. Madrid: Santillana; 2001

13. Moore I. Evaluating a materials course. Liverpool: UK Centre for Materials Education; 2003. URL: http://www.materials. ac.uk/guides/4-evaluating.pdf.

14. Kolmos A. Estrategias para desarrollar currículos basados en la formulación de problemas y organizados en base a proyectos. Educar 2004; 33: 77-96.

15. Angurel LA, Ríos R. Aprendizaje basado en proyectos aplicado a la asignatura 'Deformación y fractura de materiales de uso en ingeniería.' Zaragoza: Universidad de Zaragoza; 2006. URL: http://www.unizar.es/eees/innovacion06/comunic_publi/ bloque_II/cap_II_3.pdf.

16. Galeana de la O L. Aprendizaje basado en proyectos. Colima: Ceupromed; 2009. URL: http://ceupromed.ucol.mx/revista/ PdfArt/1/27.pdf.

17. Diez ML. Reflexiones en torno a la interculturalidad. Cuadernos de Antropología Social 2004; 19: 191-213.

18. Théodore FL, Bonvecchio-Arenas A, Blanco-García I, Carreto-Rivera Y. Representaciones sociales relacionadas con la alimentación escolar: el caso de las escuelas públicas de la Ciudad de México. Salud Colectiva 2010; 7: 215-29. 
19. Thomas JW. A review of research on project-based learning San Rafael, CA: Autodesk Foundation; 2000. URL: http:// www.bobpearlman.org/BestPractices/PBL_Research.pdf.

20. Coulby D. Intercultural education: theory and practice. Intercultural Education 2006; 17: 245-57.

21. Kripalani S, Bussey-Jones J, Katz MG, Genao I. A prescription for cultural competence in medical education. JGIM 2006; 21: 1116-20.

22. Vázquez A, Jacob I. Escribir textos académicos en la universidad: intervención didáctica y enfoques de los estudiantes. Ensayos y Experiencias 2006; 63: 53-74.

23. Vázquez A, Jacob I. La escritura y el aprendizaje en el aula universitaria: componentes cognitivos y didácticos. Innovación Educativa 2007; 7: 21-35.

24. Helle L, Tynjälä P, Olkinoura E. Project-based learning in post-secondary education -theory, practice and rubber sling shots. High Educ 2006; 51: 287-314.
25. Centre for Educational Research and Innovation. Formative assessment: improving learning in secondary classrooms. Paris: OECD; 2005.

26. Park ER, Chun MBJ, Betancourt JR, Green AR, Weissman JS. Measuring residents' perceived preparedness and skillfulness to deliver cross-cultural care. JGIM 2009; 24: 1053-6.

27. González-Peña MV, Machado-Durán MT. Extensión universitaria en la universalización de la educación superior: una mirada desde la pedagogía intercultural. Humanidades Médicas 2011; 11: 274-89.

28. Morin E. Los siete saberes necesarios para la educación del futuro. París: UNESCO; 1999.

29. Barquera S. Prevención de la diabetes mellitus: un problema mundial. Salud Publica Mex 2003; 45: 413-4. 